\title{
Mn Impurity in GaN Studied by Electron Paramagnetic Resonance
}

\author{
A. WoŁoś ${ }^{a, *}$, M. Palczewska ${ }^{b}, Z$. Wilamowski ${ }^{c}$, \\ M. Kamińska ${ }^{a}$, A. Twardowski ${ }^{a}$, M. BoćKOWSKI ${ }^{d}$, \\ I. GrzegOrY ${ }^{d}$ AND S. POROWSKI ${ }^{d}$ \\ ${ }^{a}$ Institute of Experimental Physics, Warsaw University \\ Hoża 69, 00-681 Warsaw, Poland \\ ${ }^{b}$ Institute of Electronic Materials Technology \\ Wólczyńska 133, 01-919 Warsaw, Poland \\ ${ }^{c}$ Institute of Physics, Polish Academy of Sciences \\ al. Lotników 32/46, 02-668 Warsaw, Poland \\ ${ }^{d}$ High Pressure Research Center, Polish Academy of Sciences \\ Sokołowska 29/37, 01-142 Warsaw, Poland
}

\begin{abstract}
We present the results of electron paramagnetic resonance investigations of GaN bulk crystals doped with $\mathrm{Mn}$. The EPR experiment shows the $\mathrm{Mn}^{2+}$ resonance in all the investigated $n$-type crystals, while in highly resistive samples extra doped with $\mathrm{Mg}$ acceptor the $\mathrm{Mn}^{2+}$ resonance decreases. This is a consequence of the location of $\mathrm{Mn}$ acceptor level in GaN band gap. The analysis of the spin relaxation times reveals the Korringa scattering as the dominating spin relaxation mechanism in $n$-type GaN:Mn crystals. The effective exchange constant determined from spin relaxation rate temperature dependence is of the order of $14 \mathrm{meV}$.
\end{abstract}

PACS numbers: 71.55.-i, 71.55.Eq, 71.70.Gm

\section{Introduction, experimental technique and samples}

Many experimental and theoretical efforts have been undertaken to investigate and grow room temperature ferromagnetic GaMnN [1-7]. Also the investigations of isolated $\mathrm{Mn}$ dopant in GaN became important, as the nature of $\mathrm{Mn}$ impurity in GaN decides about magnetic properties of ternary GaMnN crystals.

*corresponding author; e-mail: Agnieszka.Wolos@fuw.edu.pl 
Here we present the results of electron paramagnetic resonance (EPR) investigations of bulk GaN:Mn crystals, aiming in understanding the properties of $\mathrm{Mn}$ ion in GaN. The EPR experiments were performed using the Bruker spectrometer operating at $\mathrm{X}$-band. The spectra were collected in the temperature range of $6-50 \mathrm{~K}$.

The investigated samples were single wurzite GaN crystals grown in equilibrium high pressure technique [8]. The $\mathrm{Mn}$ content was in the range of 0.01-0.2 molar \%. We investigated both $n$-type samples with free electron concentration of the order of $10^{19} \mathrm{~cm}^{-3}$ (the residual donor in high pressure growth technique is most probably oxygen), and highly resistive GaN:Mn, Mg samples, extra doped with $\mathrm{Mg}$ acceptor.

\section{2. $\mathrm{Mn}^{2+}$ resonance in $\mathrm{GaN}: \mathrm{Mn}$ and $\mathrm{GaN}: \mathrm{Mn}, \mathrm{Mg}$}

As it was previously reported, in $n$-type GaN crystals $\mathrm{Mn}$ ion stays in ionized acceptor $\mathrm{Mn}^{2+}\left(d^{5}\right)$ configuration $[9,10]$. The EPR spectrum for that center in $n$-type GaN:Mn crystal is shown in Fig. 1. The $\mathrm{Mn}^{2+}$ resonance spectrum in

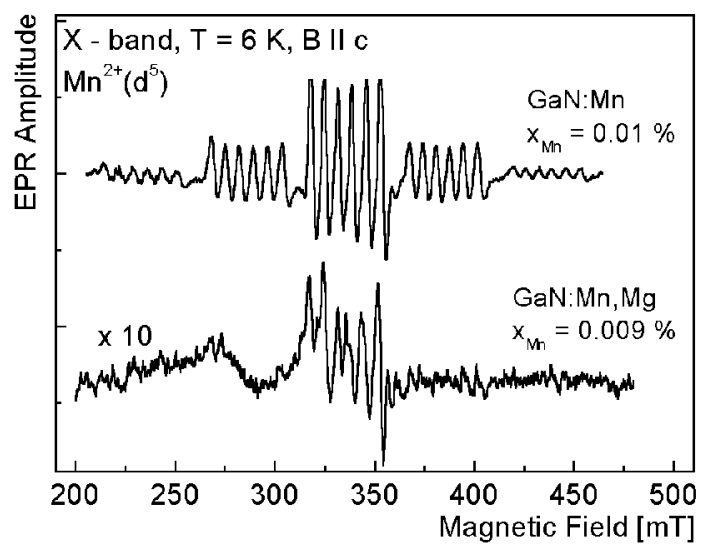

Fig. 1. EPR spectra of $\mathrm{Mn}^{2+}$ in $n$-type GaN:Mn crystal - upper curve, and in highly resistive GaN:Mn, $\mathrm{Mg}$ - lower curve. The EPR amplitude in GaN:Mn, Mg sample is about ten times lower than in $n$-type sample, even though the total Mn content in both crystals is comparable.

wurzite GaN consists of thirty lines of hyperfine structure (the spin of Mn nucleus $I=5 / 2$ ) well resolved in $B \| c$ configuration. In this configuration the lines are grouped in five bunches of fine structure for the electron spin system with spin $S=5 / 2[9-11]$.

The EPR investigations of GaN:Mn, $\mathrm{Mg}$ samples show that the $\mathrm{Mn}^{2+}$ resonance decreases for highly resistive crystals (see Fig. 1). The EPR amplitude for 
the investigated highly resistive sample is about ten times lower than for $n$-type crystal with similar Mn content. The presented result is consistent with the reported considerations about location of Mn acceptor in GaN band gap. When the Fermi level is lowered to the center of GaN energy gap (e.g. by extra doping with acceptors) the $\mathrm{Mn}$ acceptor levels are emptied from electrons and Mn stays in neutral configuration.

In order to investigate the location of Mn acceptor level in GaN a photo-EPR experiment was performed. The inset in Fig. 2 shows the EPR amplitude of the central group of $\mathrm{Mn}^{2+}$ resonance lines recorded for GaN:Mn, $\mathrm{Mg}$ sample cooled at dark, together with the signal recorded after illumination of the sample with 2.0-3.5 eV halogen lamp light. The increase in the signal intensity by a factor of two after illumination is evident. The photostimulated $\mathrm{Mn}^{2+}$ signal is metastable with barrier height for thermalization, determined from temperature dependence of the signal amplitude, equal to about $0.3 \mathrm{eV}$.

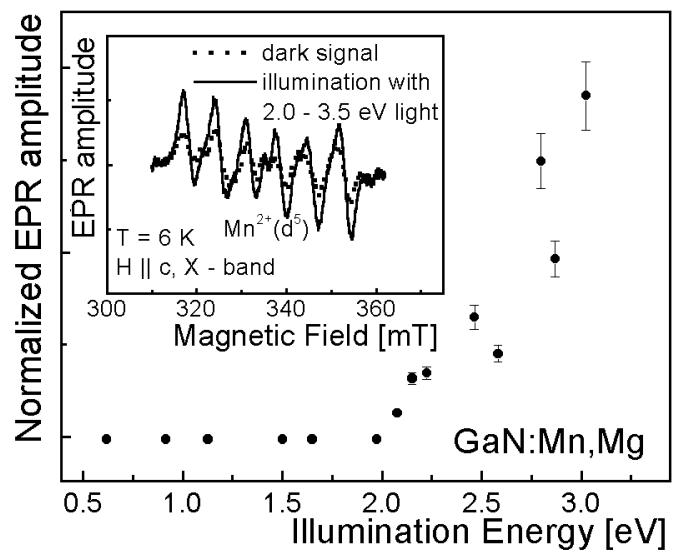

Fig. 2. Spectral dependence of the photostimulated $\mathrm{Mn}^{2+}$ resonance amplitude for $\mathrm{GaN}: \mathrm{Mn}, \mathrm{Mg}$. An onset at $2 \mathrm{eV}$ is clearly visible. Inset: central part of $\mathrm{Mn}^{2+}$ resonance before and after illumination.

The spectral dependence of the photostimulated $\mathrm{Mn}^{2+}$ amplitude is shown in Fig. 2. The data were corrected for transmission of interference filters and intensity of halogen lamp light used in the experiment. A step in the photoionization spectrum at about $2 \mathrm{eV}$ is clearly visible.

We interpret the observed photoionization as a direct process involving neutral $\mathrm{Mn}$ denoted as $\mathrm{A}^{0}$ and electrons from $\mathrm{GaN}$ valence band

$$
\mathrm{A}^{0}+h \nu \rightarrow \mathrm{Mn}^{2+}\left(d^{5}\right)+\text { hole } \mathrm{vb} .
$$

In optical absorption experiments for semi-insulating $\mathrm{GaN}$ :Mn samples a broad absorption band with an onset at about $2 \mathrm{eV}$ was reported [12]. The band 
was assigned to photoionization of the neutral Mn. Our photo-EPR experiment confirms that interpretation.

\section{Spin relaxation times, Korringa scattering mechanism}

Figure 3 shows the peak-to-peak amplitude $A_{\mathrm{pp}}$ for the central fine structure transition of $\mathrm{Mn}^{2+}$ resonance in GaN versus microwave power $P$ recorded at temperature $T=6 \mathrm{~K}$. The $A_{\mathrm{pp}}$ grows like $P^{1 / 2}$ at low microwave powers, while at high powers when the absorbed energy is comparable with the energy relaxed by the spin system, the EPR signal saturates and the $A_{\mathrm{pp}}$ decreases [13].

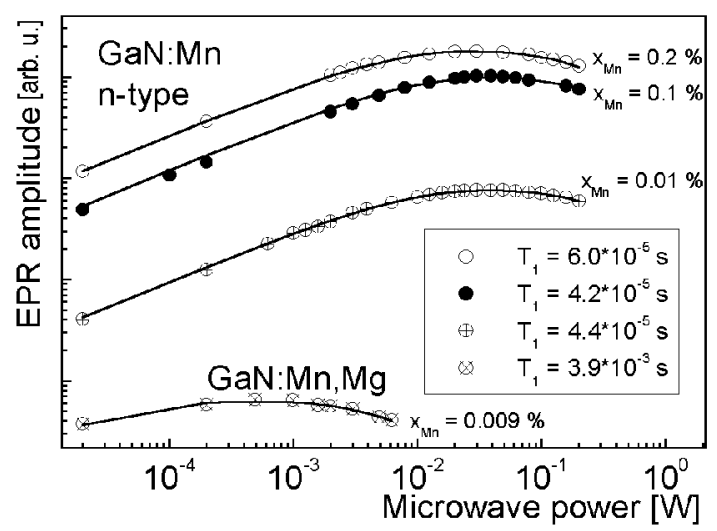

Fig. 3. $\mathrm{Mn}^{2+}$ resonance amplitude as a function of microwave power measured for GaN:Mn and GaN:Mn,Mg. Shortening of the $\mathrm{Mn}^{2+}$ relaxation time for $n$-type samples is evident. Solid lines represent a fit of the formula describing resonance amplitude vs. microwave power [13] to experimental data. The relaxation times listed in the table were determined from the fit.

The spin relaxation time $T_{1}$ is inversely proportional to the microwave power at $A_{\mathrm{pp}}$ maximum. It is clearly visible in Fig. 3 that $T_{1}$ is essentially shortened in $n$-type GaN:Mn crystals with comparison to the highly resistive $\mathrm{GaN}: \mathrm{Mn}, \mathrm{Mg}$ sample. $T_{1}$, determined precisely by the standard procedure for inhomogeneously broadened resonance lines [13], equals to $3.9 \times 10^{-3} \mathrm{~s}$ for $\mathrm{GaN}: \mathrm{Mn}, \mathrm{Mg}$, while for $n$-type GaN:Mn $T_{1}=4-6 \times 10^{-5} \mathrm{~s}$.

The relaxation rate $1 / T_{1}$ measured as a function of temperature for $n$-type GaN:Mn samples is shown in Fig. 4. The temperature dependence of the relaxation rate appears to be linear.

The essential shortening of the relaxation time in $n$-type crystals and a linear temperature dependence of relaxation rate for that group of samples point to the Korringa spin relaxation mechanism $[14,15]$ as the dominating in $n$-type GaN:Mn. 


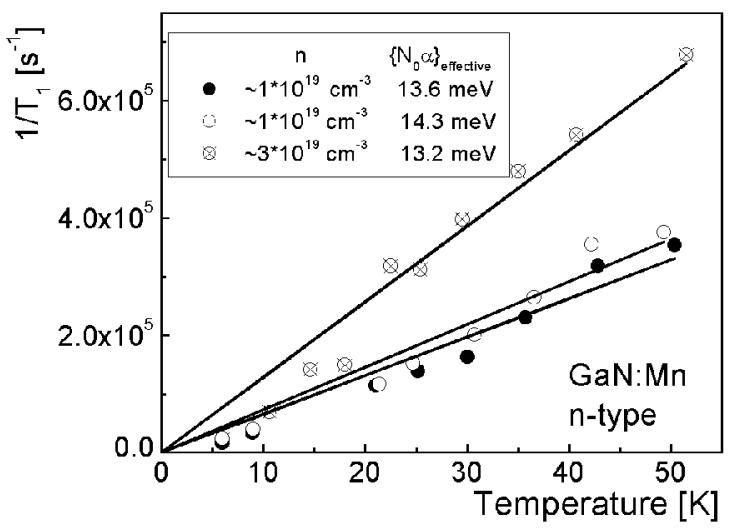

Fig. 4. $\mathrm{Mn}^{2+}$ relaxation rate versus temperature for $n$-type GaN:Mn. Mean free electron concentration and the derived effective exchange constant for each of the measured samples are listed in the Table.

Korringa spin relaxation mechanism originates from simultaneous spin-flip scattering of GaN band electrons on the localized $\mathrm{Mn}^{2+}$ center. The $\mathrm{Mn}^{2+}$ relaxation rate depends on the magnitude of the exchange interaction between band electrons spins and a localized spin of $\mathrm{Mn}^{2+}$. The formula describing temperature dependence of the relaxation rate is as follows $[14,15]$ :

$$
\frac{1}{T_{1}}=\frac{\hbar}{2 \pi}\left[\frac{5}{2}\left(\frac{5}{2}+1\right)-\left(\frac{1}{2}\right)^{2}\right]\left(N_{0} \alpha\right)^{2} \Omega_{0}^{2} \rho^{2}\left(E_{\mathrm{F}}\right) k_{\mathrm{B}} T .
$$

Here $\Omega_{0}$ is the volume of the GaN unit cell, and $N_{0}$ is defined as $1 / \Omega_{0}, \rho\left(E_{\mathrm{F}}\right)$ is the $\mathrm{GaN}$ conduction band density of states at the Fermi energy. $\alpha$ is defined as a mean value of the exchange constant $J(r)$ for conduction band electrons at the Fermi level. $J(r)$ describes exchange interaction via the Kondo Hamiltonian $H_{\mathrm{ex}}=J(r) S \sigma \delta(r)$. In the Kondo Hamiltonian $S$ is $\mathrm{Mn}^{2+}$ spin, $\sigma$ is free electron spin, $r$ is a distance between $\mathrm{Mn}$ ion and interacting free electron spin.

From the temperature dependence of the relaxation rate, assuming $\rho\left(E_{\mathrm{F}}\right)$ within the effective mass approximation with $m^{*}=0.22$, we determine the value of the effective $s-d$ exchange constant for highly $n$-type GaN:Mn $N_{0} \alpha=14 \pm 4 \mathrm{meV}$. The error in determination of the $N_{0} \alpha$ value originates mainly from the uncertainty of free electron concentration, as the samples show concentration gradient along $c$ axis of the crystal. On $\mathrm{N}$ face the concentration is more than twice higher than on Ga face [16].

\section{Conclusions}

To conclude, our EPR investigations confirmed location of Mn acceptor level in $\mathrm{GaN}$ band gap. We determined optical ionization energy from $\mathrm{GaN}$ valence band to partially empty $\mathrm{Mn}$ acceptor level equal to about $2 \mathrm{eV}$. 
The analysis of the $\mathrm{Mn}^{2+}$ relaxation times revealed the Korringa scattering as the dominating spin relaxation mechanism in highly $n$-type GaN:Mn crystals. This allowed us to determine the value of the effective $s-d$ exchange constant $N_{0} \alpha=14 \pm 4 \mathrm{meV}$, which reveals weak exchange interaction of $\mathrm{Mn}^{2+}$ with $\mathrm{GaN}$ band electrons.

\section{References}

[1] T. Dietl, H. Ohno, F. Matsukura, J. Cibert, D. Ferrand, Science 287, 1019 (2000).

[2] For a review see: T. Dietl, Phys. Status Solidi, in press.

[3] K. Sato, H. Katayama-Yoshida, Jpn. J. Appl. Phys. 40, L485 (2001).

[4] T. Sasaki, S. Sonoda, Y. Yamamoto, K. Suga, S. Shimizu, K. Kindo, H. Hori, J. Appl. Phys. 91, 7911 (2002).

[5] N. Theodoropoulou, A.F. Hebard, M.E. Overberg, C.R. Abernathy, S.J. Pearton, S.N.G. Chu, R.G. Wilson, Appl. Phys. Lett. 78, 3475 (2001).

[6] M.E. Overberg, C.R. Abernathy, S.J. Pearton, N.A. Theodoropoulou, K.T. McCarthy, A.F. Hebard, Appl. Phys. Lett. 79, 1323 (2001).

[7] M. Zajac, J. Gosk, E. Grzanka, M. Kaminska, A. Twardowski, B. Strojek, T. Szyszko, S. Podsiadlo, J. Appl. Phys. 93, 4715 (2003).

[8] S. Krukowski, M. Bockowski, B. Lucznik, I. Grzegory, S. Porowski, T. Suski, Z. Romanowski, J. Phys., Condens. Matter 13, 8881 (2001).

[9] A. Wołoś, M. Zając, J. Gosk, M. Palczewska, M. Kamińska, A. Twardowski, M. Boćkowski, I. Grzegory, S. Porowski, in: Proc. 26th ICPS "Physics of Semiconductors 2002”, Edinburgh 2002, Eds. A.R. Long, J.H. Davies, Institute of Physics, Bristol 2002, D44.

[10] T. Graf, M. Gjukic, M. Hermann, M.S. Brandt, M. Stutzmann, Phys. Rev. B 67, 165215 (2003)

[11] P.G. Baranov, I.V. Ilyin, E.N. Mokhov, A.D. Roenkov, Semicond. Sci. Technol. 11, $1843(1996)$

[12] T. Graf, M. Gjukic, M.S. Brandt, M. Stutzmann, O. Ambacher, Appl. Phys. Lett. 81, 5159 (2002).

[13] Ch.P. Poole, Electron Spin Resonance: A Comprehensive Treatise on Experimental Techniques, Wiley, New York 1967.

[14] J. Korringa, Physica XVI, 601 (1950).

[15] Z. Wilamowski, A. Mycielski, W. Jantsch, G. Hendorfer, Phys. Rev. B 38, 3621 (1988).

[16] E. Frayssinet, W. Knap, S. Krukowski, P. Perlin, P. Wisniewski, T. Suski, I. Grzegory, S. Porowski, J. Cryst. Growth 230, 442 (2001). 